\title{
On a nonparametric estimator for the finite time survival probability with zero initial surplus
}

\author{
Zhimin Zhang, Hailiang Yang, Hu Yang ${ }^{\ddagger}$
}

March 25, 2013

\begin{abstract}
In this paper, we consider the estimation of the finite time survival probability in the classical risk model when the initial surplus is zero. We construct a nonparametric estimator by Fourier inversion and kernel density estimation method. Under some mild assumptions imposed on the kernel, bandwidth and claim size density, we derive the order of the bias and variance, and show that the estimator has asymptotic normality property. Some simulation studies show that the estimator performs quite well in the finite sample setting.
\end{abstract}

Keywords: Finite time survival probability, Fourier transform, Kernel, Bias, Variance, Asymptotic normality.

\section{Introduction}

The calculation or estimation of ruin probability (or survival probability) is one of the main topics in risk theory. In this paper, we propose a nonparametric estimator for the finite time survival probability in the classical risk model, where we assume that the Poisson claim arrival intensity is known but the claim size density is unknown.

In the classical risk model, the surplus process $\left\{U_{t}, t \geq 0\right\}$ has the following form,

$$
U_{t}=u+c t-\sum_{j=1}^{N_{t}} X_{j}
$$

where $u \geq 0$ is the initial surplus, $c>0$ is the constant premium rate, $\left\{N_{t}, t \geq 0\right\}$ denoting the number of claims up to time $t$ is a Poisson process with intensity $\lambda>0$. The i.i.d. random variables

\footnotetext{
${ }^{*}$ College of Mathematics and Statistics, Chongqing University, Chongqing, 401331, PR China. Corresponding Author: Zhimin Zhang (cquzzm@163.com)

${ }^{\dagger}$ Department of Statistics and Actuarial Science, The University of Hong Kong, Pokfulam Road, Hong Kong.

${ }^{\ddagger}$ College of Mathematics and Statistics, Chongqing University, Chongqing, 401331, PR China
} 
$\left\{X_{j}, j=1,2, \cdots\right\}$, independent of $\left\{N_{t},\right\}$, have the same distribution as that of $X$ with unknown density $f$.

The ruin time defined by

$$
\tau=\inf \left\{t>0: U_{t}<0\right\}
$$

is the first time when the surplus becomes negative. One of the ruin functions of interest in ruin theory is the finite time ruin probability

$$
\psi(u, t)=\mathbb{P}\left(\tau \leq t \mid U_{0}=u\right), \quad t>0,
$$

which is the probability of ruin that occurs in $(0, t]$ when the initial surplus is $u$. Let $\varphi(u . t)=1-\psi(u, t)$ be the finite time survival probability.

The distribution of ruin time is very hard to estimate even in the classical risk model. Although some actuarial researchers have done some interesting contributions, for example, formulas for the Laplace transform of the ruin time are obtained in some cases (see e.g. Dickson and Hipp (2001)), it is still hard to obtain its distribution by inverting the Laplace transform because the parameter of the Laplace transform is implicitly embedded in the roots of the (generalized) Lundberg fundamental equation. In the classical risk model with exponential claim size distribution, an explicit formula for the distribution of the ruin time has been known for many years, see. e.g. Asmussen (2000) and Drekic and Willmot (2003). Again for exponential claim size distribution, Dickson et al. (2005) and Dickson and Borovkov (2008) obtain the density of the ruin time in the Sparre Andersen risk model with Erlang and some more general inter-claim time distributions, respectively.

Since it is difficult to study the finite time ruin probability by analytic or probabilistic method, it is necessary to study it via other approaches. In the last two decades, some researchers have done some interesting works on applications of the statistical methods in ruin theory. Various nonparametric estimators for the infinite time ruin probability are proposed by Croux and Veraverbeke (1990), Hipp (1994) and Mnatsakanov et al. (2008), etc. For the finite time ruin probability, Loisel et al. (2008, 2009) applies the empirical distribution to construct a nonparametric estimator. Statistical properties of the estimator are analyzed in detail in their papers. Recently, Qin and Pitts (2011) considers the finite time survival probability when the initial surplus is zero. They construct a plug-in estimator based on Seal's formula, and use a functional approach to study the consistency and asymptotic normality of the estimator.

In this paper, we will propose a nonparametric estimator for the finite time survival probability when the initial surplus is zero. Inspired by Qin and Pitts (2011), Seal's formula will be used to construct a plug-in estimator. Different from Qin and Pitts (2011), our estimator is based on Fourier inversion and kernel density estimate method. Note that one appealing advantage of kernel smoothing is that it can reduce the variance when an appropriate bandwidth is used. The reminder of this paper is organized as follows: In Section 2, we illustrate how to construct the plug-in type estimator in detail. In Section 3, some statistical properties of the estimator, such as the order of bias and variance, and asymptotic normality, are given. In Section 4, we present some simulation results to show the finite sample performance of the estimator. Some concluding remarks are given in Section 5. The technical proofs are given in the Appendix. 


\section{The estimator}

Throughout this paper, we will use $\varphi(t)$ to denote $\varphi(0, t)$ for notational convenience. The proposed estimator of $\varphi(t)$ is based on Seal's formula (see e.g. Seal (1978) and Gerber (1979)). Firstly, the density of the aggregate claim process $S_{t}=\sum_{j=1}^{N_{t}} X_{j}$ is given by

$$
f_{S_{t}}(y)=e^{-\lambda t} \sum_{j=0}^{\infty} \frac{(\lambda t)^{j}}{j !} f^{* j}(y), \quad y \geq 0
$$

where $f^{* 0}=\delta_{0}$ is the Dirac-Delta function at zero and $f^{* j}$ is the $j$-fold convolution of $f$ with itself for $j \geq 1$. Let

$$
g_{S_{t}}(y)=\sum_{j=1}^{\infty} \frac{(\lambda t)^{j}}{j !} f^{* j}(y)
$$

then

$$
f_{S_{t}}(y)=e^{-\lambda t} \delta_{0}(y)+e^{-\lambda t} g_{S_{t}}(y) .
$$

Seal's formula states that the finite time survival probability with zero initial surplus can be expressed as

$$
\varphi(t)=\frac{1}{c t} \int_{0}^{c t} \int_{0-}^{x} f_{S_{t}}(y) d y d x=e^{-\lambda t}+\frac{e^{-\lambda t}}{c t} \int_{0}^{c t} \int_{0}^{x} g_{S_{t}}(y) d y d x .
$$

For an integrable function $v$, we denote its Fourier transform by

$$
\phi_{v}(\omega)=\int e^{i \omega x} v(x) d x
$$

Here and after the domain of integration is the whole real line if it is not specified. If $\phi_{v}$ is integrable, then Fourier inversion transform gives

$$
v(x)=\frac{1}{2 \pi} \int e^{-i \omega x} \phi_{v}(\omega) d \omega .
$$

By some standard properties of Fourier transform, we have

$$
\phi_{g_{S_{t}}}(\omega)=\int e^{i \omega y} g_{S_{t}}(y) d y=e^{\lambda t \phi_{f}(\omega)}-1 .
$$

By Fourier inversion transform, we have

$$
g_{S_{t}}(y)=\frac{1}{2 \pi} \int e^{-i \omega y}\left(e^{\lambda t \phi_{f}(\omega)}-1\right) d \omega
$$

provide that $\left|\phi_{f}(\omega)\right|$ is integrable. We will estimate the density $g_{S_{t}}$ by replacing the characteristic function $\phi_{f}$ by some estimator. Firstly, given $n$ observations of the claim sizes $X_{1}, X_{2}, \ldots, X_{n}$, we can estimate the claim size density $f$ by the following kernel estimator

$$
\hat{f}_{n}(x)=\frac{1}{n h_{n}} \sum_{j=1}^{n} K\left(\frac{x-X_{j}}{h_{n}}\right)
$$


where $K$ is a kernel function, and $h_{n}>0$ is a positive number, usually called bandwidth, such that $h_{n} \rightarrow 0$ as $n \rightarrow \infty$. With the understanding that $h_{n}$ is a function of the sample size $n, h$ will be used in this paper for notational convenience. For more details about kernel density estimate, we refer the readers to Wand and Jones (1995). Next, we can calculate the Fourier transform of $\hat{f}_{n}$ as follows,

$$
\phi_{\hat{f}_{n}}(\omega)=\int e^{i \omega x} \frac{1}{n h} \sum_{j=1}^{n} K\left(\frac{x-X_{j}}{h}\right) d x=\phi_{e m p}(\omega) \phi_{K}(\omega h)
$$

where $\phi_{e m p}(\omega)=\frac{1}{n} \sum_{j=1}^{n} e^{i \omega X_{j}}$ is the empirical characteristic function. Finally, replacing $\phi_{f}$ in (2.2) by $\phi_{\hat{f}_{n}}$ gives the following estimator of $g_{S_{t}}(y)$,

$$
\hat{g}_{n, S_{t}}(y)=\frac{1}{2 \pi} \int e^{-i \omega y}\left(e^{\lambda t \phi_{e m p}(\omega) \phi_{K}(\omega h)}-1\right) d \omega .
$$

Now we are ready to propose our estimator for the finite time survival probability. Replacing $g_{S_{t}}(y)$ in $(2.1)$ by $\hat{g}_{n, S_{t}}(y)$ and using Fubini's theorem, we can obtain an estimator for $\varphi(t)$ as follows,

$$
\begin{aligned}
\hat{\varphi}_{n}(t) & =e^{-\lambda t}+\frac{e^{-\lambda t}}{c t} \int_{0}^{c t} \int_{0}^{x} \hat{g}_{n, S_{t}}(y) d y d x \\
& =e^{-\lambda t}+\frac{e^{-\lambda t}}{2 \pi c t} \iint_{0}^{c t} \int_{0}^{x} e^{-i \omega y}\left(e^{\lambda t \phi_{e m p}(\omega) \phi_{K}(\omega h)}-1\right) d y d x d \omega \\
& =e^{-\lambda t}+\frac{e^{-\lambda t}}{2 \pi c t} \int \frac{e^{-i \omega c t}-1+i \omega c t}{(i \omega)^{2}}\left(e^{\lambda t \phi_{e m p}(\omega) \phi_{K}(\omega h)}-1\right) d \omega .
\end{aligned}
$$

In (2.5), we can replace $\phi_{e m p}(\omega) \phi_{K}(\omega h)$ by $\phi_{f}(\omega)$ to obtain

$$
\varphi(t)=e^{-\lambda t}+\frac{e^{-\lambda t}}{2 \pi c t} \int \frac{e^{-i \omega c t}-1+i \omega c t}{(i \omega)^{2}}\left(e^{\lambda t \phi_{f}(\omega)}-1\right) d \omega .
$$

It is easy to see that the integrals in (2.5) and (2.6) are both real-valued. Formulae (2.5) and (2.6) are useful for studying the asymptotic properties of the estimator. For numerical calculation, we shall use formulae (2.1) and (2.4) because can be apply Fast Fourier (Inversion) Transform to calculate the integrands $g_{S_{t}}(y)$ and $\hat{g}_{S_{t}}(y)$. For details, see Section 4.

\section{$3 \quad$ Assumptions and asymptotic properties}

To study the estimator of the finite time survival probability, we present some assumptions imposed on the kernel $K$, bandwidth $h$ and density $f$. For a function $q$, define $\mu_{j}(q)=\int x^{j} q(x) d x, j=0,1,2, \cdots$. Also, we let $R(q)=\int q(x)^{2} d x$. Throughout this paper all the limits are taken as $n \rightarrow \infty$.

Assumption $K K$ is a probability kernel, symmetric about zero, $0<\mu_{2}(K)<\infty, R(K)<\infty$ and $\int\left|\phi_{K}(\omega)\right| d \omega<\infty$

\section{Assumption $\mathrm{H}$}


(H1) $h \rightarrow 0$;

(H2) $n h \rightarrow \infty$.

\section{Assumption F}

(F1) $f(x) \equiv 0$ for $x<0, f$ is continuously differentiable in $(0, \infty)$, right-continuous at zero, and $f^{\prime \prime}$ exists almost everywhere;

(F2) $\int\left|f^{\prime}(x)\right| d x<\infty, \int\left|f^{\prime \prime}(x)\right| d x<\infty$.

(F3) $\int\left|\phi_{f}(\omega)\right| d \omega<\infty$.

Remark 1 The assumptions $\mathbf{K}$ and $\mathbf{H}$ are standard and widely used in the nonparametric kernel estimate literature. The assumptions (F1) and (F2) are also not restrictive, and many popular densities in ruin theory, such as exponential, combination of exponentials, gamma, Pareto, satisfy these assumptions. Assumption (F3) seems to be restrictive because it excludes exponential density. However, (F3) is only a technical assumption for the study of the asymptotic properties of the estimator. As we will see in the simulation studies, our estimator also behaves well when the claim size density is exponential.

In this paper, we prefer to measure the performance of the estimator by the mean squared error (MSE),

$$
\operatorname{MSE}\left(\hat{\varphi}_{n}(t)\right)=\mathbb{E}\left(\hat{\varphi}_{n}(t)-\varphi(t)\right)^{2} .
$$

One appealing feature of MSE is the following bias-variance decomposition

$$
\operatorname{MSE}\left(\hat{\varphi}_{n}(t)\right)=\left[\operatorname{Bias}\left(\hat{\varphi}_{n}(t)\right)\right]^{2}+\operatorname{Var}\left(\hat{\varphi}_{n}(t)\right),
$$

where $\operatorname{Bias}\left(\hat{\varphi}_{n}(t)\right)=\mathbb{E} \hat{\varphi}_{n}(t)-\varphi(t)$.

Proposition 1 Suppose that assumptions $\boldsymbol{K}, \boldsymbol{H}$ and $\boldsymbol{F}$ hold. Then

$$
\operatorname{Bias}\left(\hat{\varphi}_{n}(t)\right)= \begin{cases}O\left((n h)^{-1}+h^{2}\right), & f(0)=0 \\ O\left((n h)^{-1}+h\right), & f(0)>0\end{cases}
$$

From Proposition 1 we know that the order of the bias depends on the continuity of $f$ at zero: the bias will converge to zero more quickly if the claim size density is continuous at zero. Also, from the proof of Proposition 1 we know that the order $h^{2}$ can not be improved even if the higher order derivatives of the claim size density at zero are smooth. Different from the kernel density estimate, there exists an additional term $(n h)^{-1}$ in the order of $\operatorname{Bias}\left(\hat{\varphi}_{n}(t)\right)$, which reflects the difficulty in estimating the finite time survival probability.

Remark 2 By Proposition 1 we know that the optimal bandwidth that minimizes the order of bias is given by

$$
h_{\text {bopt }}= \begin{cases}n^{-\frac{1}{3}}, & f(0)=0 \\ n^{-\frac{1}{2}}, & f(0)>0 .\end{cases}
$$


Applying the optimal bandwidth given by (3.2), we have

$$
\operatorname{Bias}\left(\hat{\varphi}_{n}(t)\right)= \begin{cases}O\left(n^{-\frac{2}{3}}\right), & f(0)=0, \\ O\left(n^{-\frac{1}{2}}\right), & f(0)>0 .\end{cases}
$$

It follows from (3.3) that the estimator depends on the smoothness of the claim size density, i.e. it would perform better if the density is continuous at zero.

Proposition 2 Suppose that Assumptions $\boldsymbol{K}, \boldsymbol{H}, \boldsymbol{F}$ hold. Then

$$
\operatorname{Var}\left(\hat{\varphi}_{n}(t)\right)= \begin{cases}O\left(n^{-1}+\left(n^{2} h\right)^{-1}+h^{4}\right), & \text { if } \int\left|\omega \phi_{f}(\omega)\right|^{4} d \omega<\infty, \\ O\left(n^{-1}+\left(n^{2} h\right)^{-1}+h^{3}\right), & \text { if }|\omega|^{3}\left|\phi_{f}(\omega)\right|^{4} d \omega<\infty, \\ O\left(n^{-1}+\left(n^{2} h\right)^{-1}+h^{2}\right), & \text { if }|\omega|^{2}\left|\phi_{f}(\omega)\right|^{4} d \omega<\infty \\ O\left(n^{-1}+\left(n^{2} h\right)^{-1}+h\right), & \text { if } \int|\omega|\left|\phi_{f}(\omega)\right|^{4} d \omega<\infty\end{cases}
$$

In particular, if one of the following additional conditions hold:

(1) $\int\left|\omega \phi_{f}(\omega)\right|^{4} d \omega<\infty, n h^{4} \rightarrow 0$;

(2) $\int|\omega|^{3}\left|\phi_{f}(\omega)\right|^{4} d \omega<\infty, n h^{3} \rightarrow 0$;

(3) $\int|\omega|^{2}\left|\phi_{f}(\omega)\right|^{4} d \omega<\infty, n h^{2} \rightarrow 0$,

then

$$
\operatorname{Var}\left(\hat{\varphi}_{n}(t)\right)=\frac{\lambda^{2} e^{-2 \lambda t}}{n c^{2}}\left(B_{0}(t)+B_{1}(t)+2 B_{2}(t)\right)+o\left(n^{-1}\right),
$$

where $B_{i}(t), i=0,1,2$, are given in Appendix $A$.

Proposition 2 shows that the order of $\operatorname{Var}\left(\hat{\varphi}_{n}(t)\right)$ depends on the smoothness of the characteristic function $\phi_{f}(\omega)$. Note that if there exists some $C, \delta, \omega_{0}>0$ such that for all $|\omega|>\omega_{0}$,

$$
\left|\phi_{f}(\omega)\right|^{4}|\omega|^{\alpha} \leq \frac{C}{1+|\omega|^{1+\delta}}, \quad \alpha=1,2,3,4,
$$

and assumptions $\boldsymbol{K}, \boldsymbol{H}, \boldsymbol{F}$, hold, then

$$
\operatorname{Var}\left(\hat{\varphi}_{n}(t)\right)=O\left(n^{-1}+\left(n^{2} h\right)^{-1}+h^{\alpha}\right) .
$$

From Proposition 2 we also know that the optimal bandwidth that minimizes the order $O\left(\left(n^{2} h\right)^{-1}+h^{\alpha}\right)$ is given by

$$
b_{v o p t}=n^{-\frac{2}{1+\alpha}}, \quad \text { if } \int|\omega|^{\alpha}\left|\phi_{f}(\omega)\right|^{4} d \omega<\infty,
$$

which is different from $h_{\text {bopt }}$ given by (3.2).

The following theorem and its corollary show the asymptotic normality of the estimator. 
Theorem 1 Suppose that assumptions $\boldsymbol{K}, \boldsymbol{H}, \boldsymbol{F}$ and one of the following assumptions hold:

(1) $\int\left|\omega \phi_{f}(\omega)\right|^{4} d \omega<\infty, n h^{4} \rightarrow 0$;

(2) $\int|\omega|^{3}\left|\phi_{f}(\omega)\right|^{4} d \omega<\infty, n h^{3} \rightarrow 0$;

(3) $\int|\omega|^{2}\left|\phi_{f}(\omega)\right|^{4} d \omega<\infty, n h^{2} \rightarrow 0$.

Then

$$
\frac{\hat{\varphi}_{n}(t)-\mathbb{E} \hat{\varphi}_{n}(t)}{\sqrt{\operatorname{Var}\left(\hat{\varphi}_{n}(t)\right)}} \stackrel{\mathfrak{D}}{\rightarrow} N(0,1),
$$

where $N(0,1)$ is the standard normal distribution.

Corollary 1 Suppose that assumptions $\boldsymbol{K}, \boldsymbol{H}, \boldsymbol{F}$ and the following assumptions hold:

$$
f(0)=0, \int\left|\omega \phi_{f}(\omega)\right|^{4} d \omega<\infty, n h^{4} \rightarrow 0, n h^{2} \rightarrow \infty
$$

Then

$$
\frac{\hat{\varphi}_{n}(t)-\varphi(t)}{\sqrt{\operatorname{Var}\left(\hat{\varphi}_{n}(t)\right)}} \stackrel{\mathfrak{D}}{\rightarrow} N(0,1) .
$$

Remark 3 We can use Corollary 1 to obtain the confidence interval and make other statistical inference for $\varphi(t)$. It is easy to see that the gamma density with shape parameter strictly larger tan 1.25 satisfies the conditions $f(0)=0$ and $\int\left|\omega \phi_{f}(\omega)\right|^{4} d \omega<\infty$.

\section{$4 \quad$ Simulation studies}

In this section we present some simulation studies to illustrate the finite sample performance of the estimator. All the results are based on formulas (2.1) and (2.4).

Firstly, we need to calculate the functions $g_{S_{t}}$ and $\hat{g}_{S_{t}}$. To this end, we use Fast Fourier (Inversion) Transform. For $g_{S_{t}}$ we have

$$
\begin{aligned}
g_{S_{t}}(y) & =\frac{1}{2 \pi} \int e^{-i \omega y}\left(e^{\lambda t \phi_{f}(\omega)}-1\right) d \omega \\
& =\frac{1}{2 \pi} \int_{0}^{\infty} e^{-i \omega y}\left(e^{\lambda t \phi_{f}(\omega)}-1\right) d \omega+\frac{1}{2 \pi} \int_{-\infty}^{0} e^{-i \omega y}\left(e^{\lambda t \phi_{f}(\omega)}-1\right) d \omega \\
& :=g_{t, 1}(y)+g_{t, 2}(y) .
\end{aligned}
$$

For a small $d>0$, set $\omega_{j}=(j-1) d, j=1,2, \ldots$ Then

$$
g_{t, 1}(y) \approx \sum_{j=1}^{N} e^{-i d y(j-1)} \frac{d\left(e^{\lambda t \phi_{f}((j-1) d)}-1\right)}{2 \pi},
$$


where $N$ is taken to be some power of 2 . In particular, for $y_{k}=2 \pi(k-1) /(d N), k=1,2, \ldots, N$, we have

$$
g_{t, 1}\left(y_{k}\right) \approx \sum_{j=1}^{N} \exp \left(-\frac{2 \pi i}{N}(j-1)(k-1)\right) \frac{d\left(e^{\lambda t \phi_{f}((j-1) d)}-1\right)}{2 \pi} .
$$

Then we can use Fast Fourier Transform to calculate $g_{t, 1}\left(y_{k}\right)$. For $g_{t, 2}$ we have

$$
\begin{aligned}
g_{t, 2}(y) & =\frac{1}{2 \pi} \int_{0}^{\infty} e^{i \omega y}\left(e^{\lambda t \phi_{f}(-\omega)}-1\right) d \omega \\
& \approx \frac{1}{N} \sum_{k=1}^{N} e^{i d y(k-1)} \frac{N d}{2 \pi}\left(e^{\lambda t \phi_{f}(-(k-1) d)}-1\right) .
\end{aligned}
$$

In particular, for $y_{j}=2 \pi(j-1) /(d N), j=1,2, \ldots, N$, we have

$$
g_{t, 2}\left(y_{j}\right) \approx \frac{1}{N} \sum_{k=1}^{N} \exp \left(\frac{2 \pi i}{N}(j-1)(k-1)\right) \frac{N d}{2 \pi}\left(e^{\lambda t \phi_{f}(-(k-1) d)}-1\right) .
$$

Then we can use Fast Fourier Inversion Transform to calculate $g_{t, 2}\left(y_{j}\right)$. By exactly the same procedure, we can calculate $\hat{g}_{S_{t}}$.

We use the Gauss kernel that is given by

$$
K(x)=\frac{1}{\sqrt{2 \pi}} e^{-\frac{x^{2}}{2}}, \quad \phi_{K}(\omega)=e^{-\frac{\omega^{2}}{2}} .
$$

For the bandwidth, we use $h_{\text {bopt }}$ that is given by (3.2). Note that $\varphi(t)$ is monotone, then it's mode is very simple. This will weaken the importance of the selection of bandwidth. The following simulation results show that the estimator performs quite well using our bandwidth selection.

As for the claim size density, we consider the following three examples.

Example 1. $\operatorname{Exp}(1)$ density with

$$
f(x)=e^{-x}, \quad x \geq 0 .
$$

Example 2. Mixture of exponentials density with

$$
f(x)=\frac{1}{3} e^{-x}+\frac{4}{3} e^{-2 x}, \quad x \geq 0 .
$$

Example 3. Gamma(6,1) density with

$$
f(x)=\frac{1}{120} x^{5} e^{-x}, \quad x \geq 0 .
$$

We set the Poisson intensity $\lambda=1$. The premium rate is chosen such that the net profit condition $c>\lambda \mathbb{E} X$ holds. For the above two examples, we set $c=1.5,1,6.5$, respectively. We calculate the approximation of the true finite time survival probability and its estimators in the time interval $[0.05,6]$ by Fast Fourier (Inversion) Transform. We set $d=0.01, N=2^{16}$. Results are given in Figures 1, 2 and 
3. In Figures 1(a), 2(a) and 3(a), we have plotted 20 estimated curves on the same figure to show the weak variability of the estimator, where the sample is 300. In Figures 1(b), 2(b) and 3(b), we plotted the bootstrap confidence bands and bootstrap means. This procedure is taken as follows, Firstly, we simulate 300 variables from exponential, mixture of exponentials and Gamma distributions; secondly, we resample 600 times from the original sample, then for fixed $t$, the we compute 600 estimated values $\hat{\varphi}_{n}(t)$; thirdly, we obtain bootstrap mean and the percentile bootstrap confidence interval from the 600 estimated values, where the confidence level is 0.95 . Compared with exponential and mixture of exponentials densities, the survival probability is easier to estimate for Gamma(6.1) density. This is due to the fact that the Fourier transform of Gamma(6,1) density has a faster convergence rate as $\omega$ tends to $\infty$.

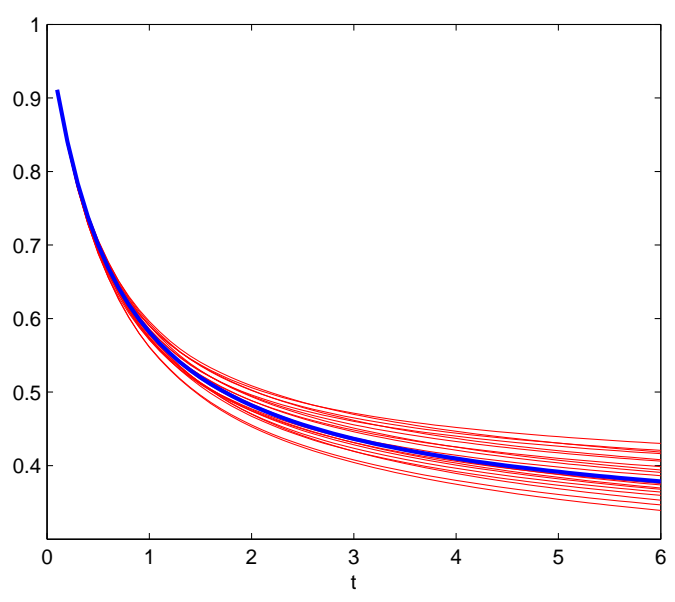

(a)

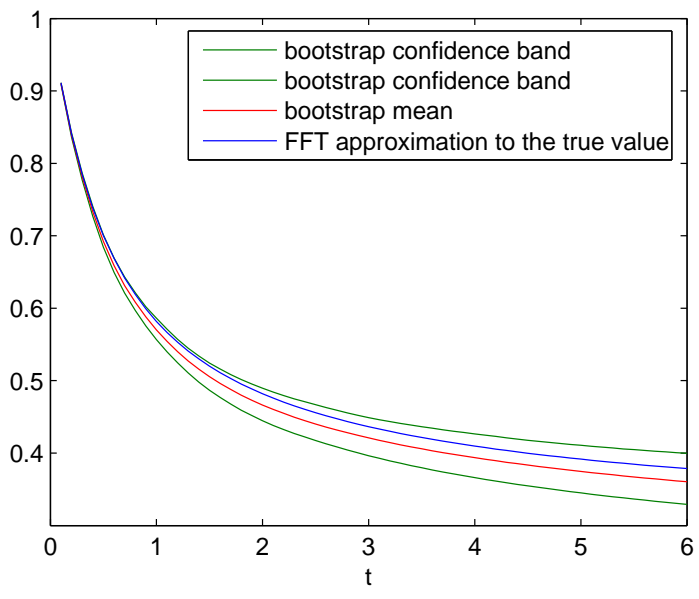

(b)

Figure 1: (a) Estimation of $\varphi(t)$ for exponential density, where the blue curve is the FFT approximation to the true value, the red curves are 20 estimate curves. (b) Bootstrap confidence band, mean, and the FFT approximation to the true value for exponential density.

\section{$5 \quad$ Concluding remarks}

In this paper, we have proposed a nonparametric estimator for the finite time survival probability in the classical risk model with zero initial surplus. The construction of the estimator is based on Seal's formula, Fourier inversion and kernel density estimate method. The order of the bias and variance are derived under some mild assumptions. We also discussed the asymptotic normality of the estimator.

All the results obtained in this paper are based on the assumption that the claim size density function is unknown. The observations of the individual claim sizes are used to construct the kernel density estimator. We can also obtain a consistent estimator when the Poisson intensity $\lambda$ is also unknown. Assume that we can observe the number of claims at some lattice time points, i.e. for $d>0$ a sample $\left\{N_{d}, N_{2 d}, N_{3 d}, \ldots,\right\}$ can be observed. Then an unbiased estimator for $\lambda$ is

$$
\hat{\lambda}_{n}=\frac{N_{n d}}{n d} .
$$

Without loss of generality, we assume that $d=1$. We can replace $\lambda$ by $\hat{\lambda}_{n}$ in (2.4) to obtain the following 


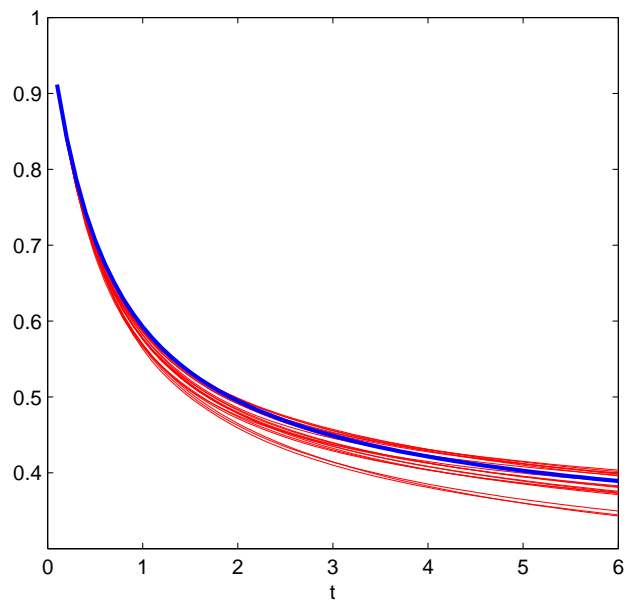

(a)

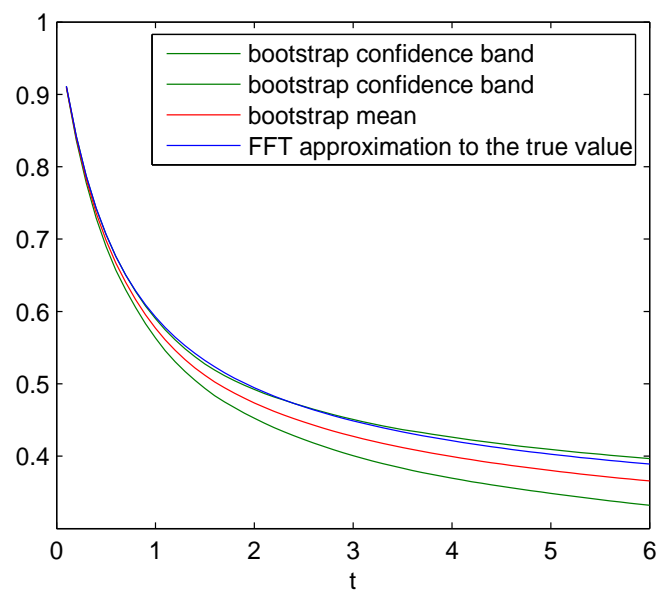

(b)

Figure 2: (a)Estimation of $\varphi(t)$ for mixture of exponentials density, where the blue curve is the FFT approximation to the true value, the red curves are 20 estimate curves. (b) Bootstrap confidence band, mean, and the FFT approximation to the true value for mixture of exponentials density.

estimator,

$$
\tilde{\varphi}_{n}(t)=e^{-\hat{\lambda}_{n} t}+\frac{e^{-\hat{\lambda}_{n} t}}{2 \pi c t} \int \frac{e^{-i \omega c t}-1+i \omega c t}{(i \omega)^{2}}\left(e^{\hat{\lambda}_{n} t \phi_{e m p}(\omega) \phi_{K}(\omega h)}-1\right) d \omega .
$$

Then by this and (2.6) we have

$$
\begin{aligned}
\tilde{\varphi}_{n}(t)-\varphi(t)= & \left(e^{-\hat{\lambda}_{n} t}-e^{-\lambda t}\right)\left(1-\frac{1}{2 \pi c t} \int \frac{e^{-i \omega c t}-1+i \omega c t}{(i \omega)^{2}} d \omega\right)+\hat{\varphi}_{n}(t)-\varphi(t) \\
& +\frac{1}{2 \pi c t} \int \frac{e^{-i \omega c t}-1+i \omega c t}{(i \omega)^{2}}\left(e^{-\hat{\lambda}_{n} t\left(1-\phi_{e m p}(\omega) \phi_{K}(\omega h)\right)}-e^{-\lambda t\left(1-\phi_{e m p}(\omega) \phi_{K}(\omega h)\right)}\right) d \omega .
\end{aligned}
$$

It is easy to see from the above formula that $\tilde{\varphi}_{n}(t)$ is a consistent estimator under assumptions $\boldsymbol{K}, \boldsymbol{H}, \boldsymbol{F}$. However, more tedious arguments are called for analyzing the convergence rate.

\section{Acknowledgements}

The authors would like to express their sincere thanks to an anonymous referee for his/her comments and suggestions, which substantially improved the paper. The first author acknowledges the support from National Natural Science Foundation of China (project No. 11101451) and the Fundamental Research Funds for the Central Universities (project No. CDJRC11100001).

\section{References}

[1] Asmussen, S., 2000. Ruin Probabilities. World Scientific, Singapore.

[2] Borovkov, B.A., Dickson, D.C.M., 2008. On the ruin time distribution for a Sparre Andersen process with exponential claim sizes. Insurance: Mathematics and Economics 42, 1104-1108 


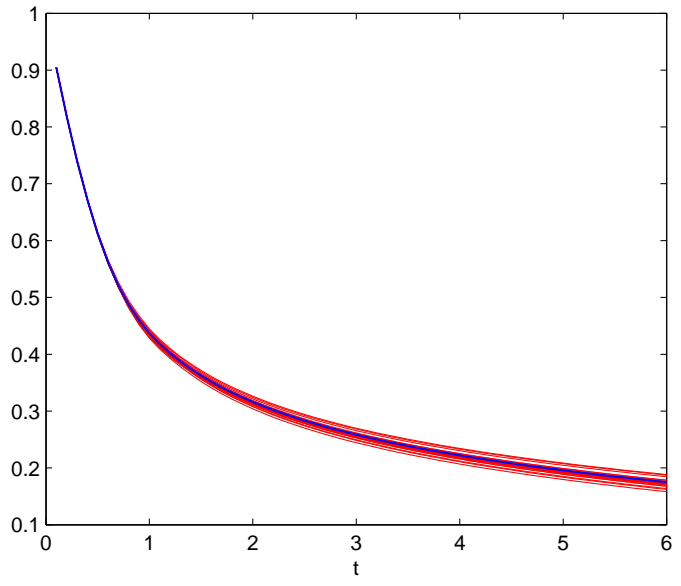

(a)

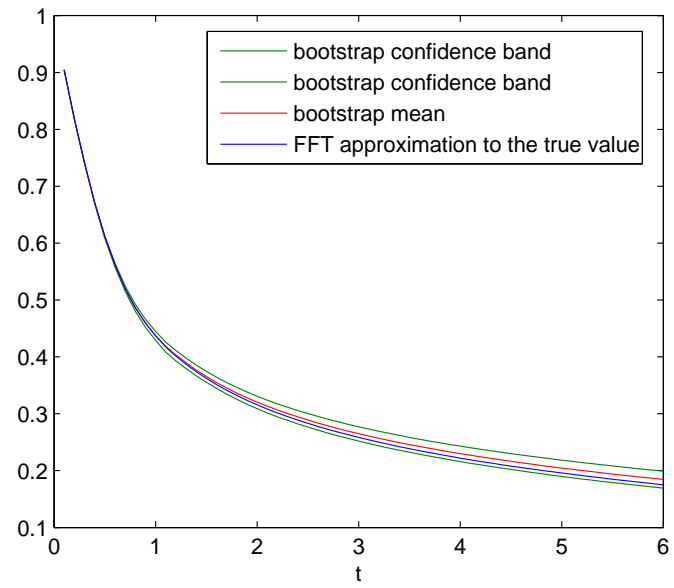

(b)

Figure 3: (a) Estimation of $\varphi(t)$ for Gamma(6,1) density, where the blue curve is the FFT approximation to the true value, the red curves are 20 estimate curves. (b) Bootstrap confidence band, mean, and the FFT approximation to the true value for Gamma(6,1) density.

[3] Croux, K., Veraverbeke, N., 1990. Nonparametric estimators for the probability of ruin. Insurance: Mathematics and Economics 9, 127-130.

[4] Dickson, D.C.M., Hipp, C., 2001. On the time to ruin for $\operatorname{Erlang(2)~risk~process.~Insurance:~Mathe-~}$ matics and Economics 29, 333-344.

[5] Dickson, D.C.M., Hughes, B.D., Zhang, L., 2005. The density of the time to ruin for a Sparre Andersen process with Erlang arrivals and exponential claims. Scandinavian Actuarial Journal 358376.

[6] Drekic, S., Willmot, G.E., 2003. On the density and moments of the time of ruin with exponential claims. ASTIN Bulletin 33, 11-21.

[7] Gerber, H.U., 1979. An introduction to mathematical risk theory. University of Pennsylvania, Philadelphia.

[8] Hall, P., Heyde, C.C., 1980. Martingale Limit Theory and Its Application. Academic Press.

[9] Hipp, C., 1994. Estimators and bootstrap confidence intervals for ruin probabilities. ASTIN Bulletin $19,57-70$.

[10] Loisel, S., Mazza, C., Rullière, D., 2008. Robustness analysis and convergence of empirical finite-time ruin probabilities and estimation risk solvency margin. Insurance: Mathematics and Economics 42, 746-762.

[11] Loisel, S., Mazza, C., Rullière, D., 2009. Convergence and asymptotic variance of bootstrapped finitetime ruin probabilities with partly shifted risk processes. Insurance: Mathematics and Economics $45,374-381$.

[12] Mnatsakanov, R., Ruymgaart, L.L., Ruymgaart, F.H., 2008. Nonparametric estimation of ruin probabilities given a random sample of claims. Mathematical Methods of Statistics 17, 35-43. 
[13] Qin, L., Pitts, S.M., 2011. Nonparametric estimation of the finite-time survival probability with zero initial capital in the classical risk model. Methodologh and Computing in Applied Probability, in press.

[14] Seal, H., 1978. Survival probabilities: the goal of risk theory. Wiley, Chichester.

[15] van Eeden, C., 1985. Mean integrated squared error of kernel estimators when the density and its derivative are not necessarily continuous. Annals of Institute of Statistical Mathematics 37, 461-472.

[16] Wand, M.P., Jones, M.C., 1995. Kernel Smoothing, Chapman \& Hall, London.

\section{Appendix}

\section{A Proofs of the results in Section 3}

By (2.5) and (2.6), we have

$$
\begin{aligned}
\hat{\varphi}_{n}(t)-\varphi(t)= & \frac{e^{-\lambda t}}{2 \pi c t} \int \frac{e^{-i \omega c t}-1+i \omega c t}{(i \omega)^{2}}\left(e^{\lambda t \phi_{e m p}(\omega) \phi_{K}(\omega h)}-e^{\lambda t \phi_{f}(\omega)}\right) d \omega \\
= & \frac{e^{-\lambda t}}{2 \pi c t} \int \frac{e^{-i \omega c t}-1+i \omega c t}{(i \omega)^{2}} e^{\lambda t \phi_{f}(\omega)}\left(e^{\lambda t\left(\phi_{e m p}(\omega) \phi_{K}(\omega h)-\phi_{f}(\omega)\right)}-1\right) d \omega \\
= & \frac{\lambda e^{-\lambda t}}{2 \pi c} \int \frac{e^{-i \omega c t}-1+i \omega c t}{(i \omega)^{2}} e^{\lambda t \phi_{f}(\omega)}\left(\phi_{e m p}(\omega) \phi_{K}(\omega h)-\phi_{f}(\omega)\right) d \omega \\
& +\frac{e^{-\lambda t}}{2 \pi c t} \int \frac{e^{-i \omega c t}-1+i \omega c t}{(i \omega)^{2}} e^{\lambda t \phi_{f}(\omega)} L_{n}(\omega) d \omega \\
= & \mathrm{I}+\mathrm{II},
\end{aligned}
$$

where

$$
L_{n}(\omega)=e^{\lambda t\left(\phi_{e m p}(\omega) \phi_{K}(\omega h)-\phi_{f}(\omega)\right)}-1-\lambda t\left(\phi_{e m p}(\omega) \phi_{K}(\omega h)-\phi_{f}(\omega)\right)
$$

In the sequel, we will use $C$ to denote a finite generic constant that is possibly dependent on the parameters $c, \lambda, t$, and can take different values at different steps. In order to study the order of the bias and variance, we need the following Taylor's expansions which are special cases of Lemma 4.3 and Lemma 4.4 in van Eeden (1985).

Lemma 1 Suppose that assumption $\boldsymbol{F}$ holds. If $x>0$ and $x-$ uh $<0$, then

$$
f(x-u h)-f(x)+u h f^{\prime}(x)-h^{2} \int_{0}^{u}(u-s) f^{\prime \prime}(x-s h) d s=-f^{\prime}(0+)(x-u h)-f(0) .
$$

If $x<0$ and $x-u h>0$, then

$$
f(x-u h)-f(x)+u h f^{\prime}(x)-h^{2} \int_{0}^{u}(u-s) f^{\prime \prime}(x-s h) d s=f^{\prime}(0+)(x-u h)+f(0) .
$$


If $x(x-u h)>0$, then

$$
f(x-u h)-f(x)+u h f^{\prime}(x)-h^{2} \int_{0}^{u}(u-s) f^{\prime \prime}(x-s h) d s=0 .
$$

Lemma 2 Suppose that assumptions $\boldsymbol{K}, \boldsymbol{F}$ and (H1) hold. Then

$$
\mathbb{E} \int_{0}^{c t} \int_{0}^{x}\left(\hat{f}_{n}(y)-f(y)\right) d y d x= \begin{cases}O\left(h^{2}\right), & f(0)=0 \\ O(h), & f(0)>0\end{cases}
$$

Proof. Note that $f(x) \equiv 0$ for $x<0$. Using the Taylor's expansions given in Lemma 1, we have

$$
\begin{aligned}
& \mathbb{E} \int_{0}^{c t} \int_{0}^{x}\left(\hat{f}_{n}(y)-f(y)\right) d y d x \\
= & \int_{0}^{c t} \int_{0}^{x} \int K(u)[f(y-u h)-f(y)] d u d y d x \\
= & h^{2} \int_{0}^{c t} \int_{0}^{x} \iint_{0}^{u} K(u)(u-s) f^{\prime \prime}(y-s h) d s d u d y d x \\
& -\int_{0}^{c t} \int_{0}^{x} \int 1_{(y-u h<0)} K(u)\left(f^{\prime}(0+)(y-u h)+f(0)\right) d u d y d x .
\end{aligned}
$$

By Fubini's theorem,

$$
\begin{aligned}
& \left|\int_{0}^{c t} \int_{0}^{x} \iint_{0}^{u} K(u)(u-s) f^{\prime \prime}(y-s h) d s d u d y d x\right| \\
= & \left|\iint_{0}^{u} \int_{0}^{c t} \int_{0}^{x} K(u)(u-s) f^{\prime \prime}(y-s h) d y d x d s d u\right| \leq \frac{1}{2} c t \mu_{2}(K) \int\left|f^{\prime \prime}(y)\right| d y .
\end{aligned}
$$

By Fubini's theorem again we have

$$
\begin{aligned}
& \int_{0}^{c t} \int_{0}^{x} \int_{(y<u h)} K(u)(y-u h) d u d y d x \\
= & \int_{0}^{\infty} \int_{0}^{c t} \int_{0}^{x \wedge u h} K(u)(y-u h) d y d x d u \\
= & \int_{0}^{\frac{c t}{h}} K(u)\left(\int_{0}^{u h} \int_{0}^{x}(y-u h) d y d x+\int_{u h}^{c t} \int_{0}^{u h}(y-u h) d y d x\right) d u \\
& +\int_{\frac{c t}{h}}^{\infty} K(u) \int_{0}^{c t} \int_{0}^{x}(y-u h) d y d x d u \\
= & \int_{0}^{\frac{c t}{h}} K(u)\left(\frac{1}{6} u^{3} h^{3}-\frac{1}{2} c t u^{2} h^{2}\right) d u+\int_{\frac{c t}{h}}^{\infty} K(u)\left(\frac{1}{6} c^{3} t^{3}-\frac{1}{2} c^{2} t^{2} u h\right) d u .
\end{aligned}
$$


Then using the following results

$$
\begin{aligned}
& \int_{0}^{\frac{c t}{h}} K(u) u^{2} h^{2} d u<h^{2} \mu_{2}(K), \\
& \int_{0}^{\frac{c t}{h}} K(u) u^{3} h^{3} d u<h^{2} c t \int_{0}^{\frac{c t}{h}} K(u) u^{2} d u<h^{2} c t \mu_{2}(K), \\
& \int_{\frac{c t}{h}}^{\infty} K(u) d u<\frac{h^{2}}{c^{2} t^{2}} \int_{\frac{c t}{h}}^{\infty} K(u) u^{2} d u<h^{2} \frac{\mu_{2}(K)}{c^{2} t^{2}} \\
& \int_{\frac{c t}{h}}^{\infty} K(u) u h d u<\frac{h^{2}}{c t} \int_{\frac{c t}{h}}^{\infty} K(u) u^{2} d u<h^{2} \frac{\mu_{2}(K)}{c t}
\end{aligned}
$$

we obtain

$$
\int_{0}^{c t} \int_{0}^{x} \int_{(y<u h)} K(u)(y-u h) d u d y d x=O\left(h^{2}\right) .
$$

Similarly, we can show that

$$
\int_{0}^{c t} \int_{0}^{x} \int_{(y<u h)} K(u) d u d y d x=O(h) .
$$

By (A.2-A.5), we complete the proof.

Lemma 3 Suppose that assumptions $\boldsymbol{K}, \boldsymbol{F}$ and (H1) hold. Then for $m=1,2, \cdots$,

$$
\mathbb{E} \int_{0}^{c t} \int_{0}^{x} f^{* m} *\left(\hat{f}_{n}-f\right)(y) d y d x= \begin{cases}O\left(h^{2}\right), & f(0)=0 \\ O(h), & f(0)>0\end{cases}
$$

Proof. Similar to Lemma 2, we can use the Taylor's expansions given in Lemma 1 to obtain

$$
\begin{aligned}
& \mathbb{E} \int_{0}^{c t} \int_{0}^{x} f^{* m} *\left(\hat{f}_{n}-f\right)(y) d y d x \\
= & \int_{0}^{c t} \int_{0}^{x} \iint f^{* m}(y-z) K(u)(f(z-u h)-f(z)) d u d z d y d x \\
= & h^{2} \int_{0}^{c t} \int_{0}^{x} \iiint_{0}^{u} f^{* m}(y-z) K(u)(u-s) f^{\prime \prime}(z-s h) d s d u d z d y d x \\
& +\int_{0}^{c t} \int_{0}^{x} \iint 1_{(z<0, z-u h>0)} f^{* m}(y-z) K(u)\left[f^{\prime}(0+)(z-u h)+f(0)\right] d u d z d y d x \\
& -\int_{0}^{c t} \int_{0}^{x} \iint 1_{(z>0, z-u h<0)} f^{* m}(y-z) K(u)\left[f^{\prime}(0+)(z-u h)+f(0)\right] d u d z d y d x .
\end{aligned}
$$

By Fubini's theorem, we have

$$
\begin{aligned}
& \left|\int_{0}^{c t} \int_{0}^{x} \iiint_{0}^{u} f^{* m}(y-z) K(u)(u-s) f^{\prime \prime}(z-s h) d s d u d z d y d x\right| \\
= & \left|\iint_{0}^{u} \iint_{0}^{c t} \int_{0}^{x} f^{* m}(y-z) K(u)(u-s) f^{\prime \prime}(z-s h) d y d x d z d s d u\right| \\
\leq & \frac{1}{2} c t \mu_{2}(K) \int\left|f^{\prime \prime}(x)\right| d x .
\end{aligned}
$$


Since the density $f^{* m}$ is bounded, then

$$
\begin{aligned}
& \int_{0}^{c t} \int_{0}^{x} \iint 1_{(z<0, z-u h>0)} f^{* m}(y-z) K(u)(z-u h) d u d z d y d x \\
\leq & C \int_{0}^{c t} \int_{0}^{x} \int_{-\infty}^{0} \int_{u h}^{0} K(u)(z-u h) d z d u d y d x \\
= & \frac{C}{8} c^{2} t^{2} \mu_{2}(K) h^{2} .
\end{aligned}
$$

Similarly, we can obtain

$$
\begin{gathered}
\int_{0}^{c t} \int_{0}^{x} \iint 1_{(z>0, z-u h<0)} f^{* m}(y-z) K(u)(u h-z) d u d z d y d x=O\left(h^{2}\right), \\
\int_{0}^{c t} \int_{0}^{x} \iint 1_{(z<0, z-u h>0)} f^{* m}(y-z) K(u) d u d z d y d x=O(h),
\end{gathered}
$$

and

$$
\int_{0}^{c t} \int_{0}^{x} \iint 1_{(z>0, z-u h<0)} f^{* m}(y-z) K(u) d u d z d y d x=O(h) .
$$

Combining above results completes the proof.

The following lemma is due to Theorem 3.1 in van Eeden (1985).

Lemma 4 Suppose that assumptions $\boldsymbol{K}, \boldsymbol{F}$ and $\boldsymbol{H}$ hold. Then the mean integrated squared error (MISE) of $\hat{f}_{n}$ is given by

$$
\operatorname{MISE}\left(\hat{f}_{n}\right)= \begin{cases}O\left((n h)^{-1}+h^{4}\right), & f(0)=f^{\prime}(0+)=0, \\ O\left((n h)^{-1}+h^{3}\right), & f(0)=0, f^{\prime}(0+) \neq 0 \\ O\left((n h)^{-1}+h\right), & f(0)>0\end{cases}
$$

Proof of Proposition 1. By (A.1), we have

$$
\operatorname{Bias}\left(\hat{\varphi}_{n}(t)\right)=\mathbb{E} \mathrm{I}+\mathbb{E} \mathrm{II} .
$$

From the derivation procedure of $(2.5)$, we have

$$
\begin{aligned}
\mathrm{I} & =\frac{\lambda e^{-\lambda t}}{2 \pi c} \iint_{0}^{c t} \int_{0}^{x} e^{-i \omega y} e^{\lambda t \phi_{f}(\omega)}\left(\phi_{e m p}(\omega) \phi_{K}(\omega)-\phi_{f}(\omega)\right) d y d x d \omega \\
& =\frac{\lambda e^{-\lambda t}}{c} \sum_{m=0}^{\infty} \frac{(\lambda t)^{m}}{m !} \int_{0}^{c t} \int_{0}^{x} f^{* m} *\left(\hat{f}_{n}-f\right)(y) d y d x .
\end{aligned}
$$

Thus, by Lemma 2 and Lemma 3 we have

$$
\mathbb{E} \mathrm{I}= \begin{cases}O\left(h^{2}\right), & f(0)=0, \\ O(h), & f(0)>0 .\end{cases}
$$


Now we consider the order of $\mathbb{E} I$ I. Since $\left|\phi_{\text {emp }}(\omega) \phi_{K}(\omega h)-\phi_{f}(\omega)\right|<2$, it is easy to see that there exists some constant $C$ such that the following inequality holds uniformly in $\omega$,

$$
\left|L_{n}(\omega)\right| \leq C\left|\phi_{e m p}(\omega) \phi_{K}(\omega h)-\phi_{f}(\omega)\right|^{2},
$$

which together with the following inequalities

$$
\left|e^{-i \omega c t}-1+i \omega c t\right| \leq \frac{1}{2}|i \omega c t|^{2}, \quad\left|e^{\lambda t \phi_{f}(\omega)}\right| \leq e^{\lambda t},
$$

gives

$$
\begin{aligned}
|\mathbb{E I I}| & \leq \frac{C}{2 \pi} \mathbb{E} \int\left|\phi_{\text {emp }}(\omega) \phi_{K}(\omega h)-\phi_{f}(\omega)\right|^{2} d \omega \\
& =C \cdot \mathbb{E} \int\left(\hat{f}_{n}(x)-f(x)\right)^{2} d x \\
& =C \cdot \operatorname{MISE}\left(\hat{f}_{n}\right) .
\end{aligned}
$$

where we have used Parseval's identity in the second step. Thus, by Lemma 4 we obtain

$$
\mathbb{E} \mathrm{II}= \begin{cases}O\left((n h)^{-1}+h^{4}\right), & f(0)=f^{\prime}(0+)=0, \\ O\left((n h)^{-1}+h^{3}\right), & f(0)=0, f^{\prime}(0+) \neq 0, \\ O\left((n h)^{-1}+h\right), & f(0)>0 .\end{cases}
$$

Finally, combining (A.6) and (A.9) completes the proof.

In order to find the order of $\operatorname{Var}\left(\hat{\varphi}_{n}(t)\right)$, we need two lemmas.

Lemma 5 Suppose that assumptions $\boldsymbol{K}, \boldsymbol{F}$ and (H1) hold. Then

$$
\begin{aligned}
& \operatorname{Var}\left(\int_{0}^{c t} \int_{0}^{x} \hat{f}_{n}(y) d y d x\right)=\frac{1}{n} B_{0}(t)+o\left(n^{-1}\right), \\
& \operatorname{Var}\left(\int_{0}^{c t} \int_{0}^{x} g_{S_{t}} * \hat{f}_{n}(y) d y d x\right)=\frac{1}{n} B_{1}(t)+o\left(n^{-1}\right), \\
& \operatorname{Cov}\left(\int_{0}^{c t} \int_{0}^{x} \hat{f}_{n}(y) d y d x, \int_{0}^{c t} \int_{0}^{x} g_{S_{t}} * \hat{f}_{n}(y) d y d x\right)=\frac{1}{n} B_{2}(t)+o\left(n^{-1}\right),
\end{aligned}
$$

where

$$
\begin{aligned}
B_{0}(t)= & \int_{0}^{c t} \int_{0}^{c t} \int_{0}^{x_{1} \wedge x_{2}} f(y) d y d x_{2} d x_{1}-\left(\int_{0}^{c t} \int_{0}^{x} f(y) d y d x\right)^{2} \\
B_{1}(t)= & \int_{0}^{c t} \int_{0}^{c t} \int_{0}^{\infty} \int_{0}^{x_{1}} \int_{0}^{x_{2}} g_{S_{t}}\left(y_{1}-z\right) g_{S_{t}}\left(y_{2}-z\right) f(z) \mathbf{1}_{\left(y_{1}>z, y_{2}>z\right)} d y_{2} d y_{1} d z d x_{2} d x_{1} \\
& -\left(\int_{0}^{c t} \int_{0}^{x} g_{S_{t}} * f(y) d y d x\right)^{2} \\
B_{2}(t)= & \int_{0}^{c t} \int_{0}^{c t} \int_{0}^{x_{1}} \int_{0}^{x_{2}} g_{S_{t}}\left(y_{2}-y_{1}\right) f\left(y_{1}\right) \mathbf{1}_{\left(y_{2}>y_{1}\right)} d y_{2} d y_{1} d x_{2} d x_{1} \\
& -\left(\int_{0}^{c t} \int_{0}^{x} f(y) d y d x\right)\left(\int_{0}^{c t} \int_{0}^{x} g_{S_{t}} * f(y) d y d x\right)
\end{aligned}
$$


Proof. We only prove (A.12) since (A.10) and (A.11) can be obtained in a similar way. Let $K_{h}(\cdot)=$ $\frac{1}{h} K(\cdot / h)$. By Lemma 2 and Lemma 3, we know that

$$
\begin{aligned}
& \mathbb{E} \int_{0}^{c t} \int_{0}^{x} \hat{f}_{n}(y) d y d x=\int_{0}^{c t} \int_{0}^{x} f(y) d y d x+o(1), \\
& \mathbb{E} \int_{0}^{c t} \int_{0}^{x} g_{S_{t}} * \hat{f}_{n}(y) d y d x=\int_{0}^{c t} \int_{0}^{x} g_{S_{t}} * f(y) d y d x+o(1) .
\end{aligned}
$$

Straightforward calculation gives

$$
\begin{aligned}
& \operatorname{Cov}\left(\int_{0}^{c t} \int_{0}^{x} \hat{f}_{n}(y) d y d x, \int_{0}^{c t} \int_{0}^{x} g_{S_{t}} * \hat{f}_{n}(y) d y d x\right) \\
= & \frac{1}{n} \mathbb{E}\left[\int_{0}^{c t} \int_{0}^{x_{1}} K_{h}\left(y_{1}-X\right) d y_{1} d x_{1} \int_{0}^{c t} \int_{0}^{x_{2}} \int g_{S_{t}}\left(y_{2}-z\right) K_{h}(z-X) d z d y_{2} d x_{2}\right] \\
& -\frac{1}{n}\left(\int_{0}^{c t} \int_{0}^{x} f(y) d y d x\right)\left(\int_{0}^{c t} \int_{0}^{x} g_{S_{t}} * f(y) d y d x\right)+o\left(n^{-1}\right) .
\end{aligned}
$$

With the understanding that $f(x)=g_{S_{t}}(x)=0$ for $x<0$, we can calculate the expectation in the above equation as follows,

$$
\begin{aligned}
& \mathbb{E} \int_{0}^{c t} \int_{0}^{x_{1}} \int_{0}^{c t} \int_{0}^{x_{2}} \int K_{h}\left(y_{1}-X\right) K_{h}(z-X) g_{S_{t}}\left(y_{2}-z\right) d z d y_{2} d x_{2} d y_{1} d x_{1} \\
= & \iint_{0}^{c t} \int_{0}^{x_{1}} \int_{0}^{c t} \int_{0}^{x_{2}} \int K_{h}\left(y_{1}-s\right) K_{h}(z-s) g_{S_{t}}\left(y_{2}-z\right) f(s) d z d y_{2} d x_{2} d y_{1} d x_{1} d s \\
= & \iint_{0}^{c t} \int_{0}^{x_{1}} \int_{0}^{c t} \int_{0}^{x_{2}} \int K\left(u_{1}\right) K_{h}\left(z-y_{1}+u_{1} h\right) g_{S_{t}}\left(y_{2}-z\right) f\left(y_{1}-u_{1} h\right) d z d y_{2} d x_{2} d y_{1} d x_{1} d u_{1} \\
= & \iint_{0}^{c t} \int_{0}^{x_{1}} \int_{0}^{c t} \int_{0}^{x_{2}} \int K\left(u_{1}\right) K\left(u_{2}\right) g_{S_{t}}\left(y_{2}-y_{1}-\left(u_{2}-u_{1}\right) h\right) f\left(y_{1}-u_{1} h\right) d u_{2} d y_{2} d x_{2} d y_{1} d x_{1} d u_{1} \\
= & \iiint_{0}^{c t} \int_{0}^{x_{1}} \int_{0}^{c t} \int_{0}^{x_{2}} \int K\left(u_{1}\right) K\left(u_{2}\right) g_{S_{t}}\left(y_{2}-y_{1}-\left(u_{2}-u_{1}\right) h\right) f\left(y_{1}-u_{1} h\right) d y_{2} d x_{2} d y_{1} d x_{1} d u_{2} d u_{1} \\
= & \int_{0}^{c t} \int_{0}^{c t} \int_{0}^{x_{1}} \int_{0}^{x_{2}} g_{S_{t}}\left(y_{2}-y_{1}\right) f\left(y_{1}\right) d y_{2} d y_{1} d x_{2} d x_{1}+o(1),
\end{aligned}
$$

where the second and third steps follow from changing variables $u_{1}=\frac{y_{1}-s}{h}$ and $u_{2}=\frac{z-y_{1}}{h}+u_{1}$, and the last two steps follow by using Fubini's theorem, dominated convergence theorem and the identity $\iint K\left(u_{1}\right) K\left(u_{2}\right) d u_{1} d u_{2}=1$. This completes the proof.

Lemma 6 Suppose that assumptions $\boldsymbol{K}, \boldsymbol{H}, \boldsymbol{F}$ hold. Then

$$
\begin{gathered}
\operatorname{Var}\left(\int \frac{e^{-i \omega c t}-1+i \omega c t}{(i \omega)^{2}} e^{\lambda t \phi_{f}(\omega)} L_{n}(\omega) d \omega\right) \\
= \begin{cases}O\left(\left(n^{2} h\right)^{-1}+h^{4}\right), & \text { if } \int\left|\omega \phi_{f}(\omega)\right|^{4} d \omega<\infty, \\
O\left(\left(n^{2} h\right)^{-1}+h^{3}\right), & \text { if } \int|\omega|^{3}\left|\phi_{f}(\omega)\right|^{4} d \omega<\infty, \\
O\left(\left(n^{2} h\right)^{-1}+h^{2}\right), & \text { if } \int|\omega|^{2}\left|\phi_{f}(\omega)\right|^{4} d \omega<\infty, \\
O\left(\left(n^{2} h\right)^{-1}+h\right), & \text { if } \int|\omega|\left|\phi_{f}(\omega)\right|^{4} d \omega<\infty .\end{cases}
\end{gathered}
$$


Proof. According to the inequalities (A.7), (A.8) and Cauchy-Schwarz inequality, we have

$$
\begin{aligned}
& \operatorname{Var}\left(\int \frac{e^{-i \omega c t}-1+i \omega c t}{(i \omega)^{2}} e^{\lambda t \phi_{f}(\omega)} L_{n}(\omega) d \omega\right) \\
\leq & \mathbb{E}\left|\int \frac{e^{-i \omega c t}-1+i \omega c t}{(i \omega)^{2}} e^{\lambda t \phi_{f}(\omega)} L_{n}(\omega) d \omega\right|^{2} \\
\leq & C \cdot \mathbb{E}\left(\int\left|\frac{e^{-i \omega c t}-1+i \omega c t}{(i \omega)^{2}}\left(\phi_{e m p}(\omega) \phi_{K}(\omega h)-\phi_{f}(\omega)\right)^{2}\right| d \omega\right)^{2} \\
\leq & C\left(\int\left|\frac{e^{-i \omega c t}-1+i \omega c t}{(i \omega)^{2}}\right|^{2} d \omega\right) \mathbb{E} \int\left|\phi_{e m p}(\omega) \phi_{K}(\omega h)-\phi_{f}(\omega)\right|^{4} d \omega, \\
\leq & C \cdot \mathbb{E} \int\left|\phi_{e m p}(\omega) \phi_{K}(\omega h)-\phi_{f}(\omega)\right|^{4} d \omega,
\end{aligned}
$$

where the last step follows from the fact that

$$
\int\left|\frac{e^{-i \omega c t}-1+i \omega c t}{(i \omega)^{2}}\right|^{2} d \omega<\infty .
$$

Using $C_{r}$-inequality, we have

$$
\begin{aligned}
& \mathbb{E} \int\left|\phi_{e m p}(\omega) \phi_{K}(\omega h)-\phi_{f}(\omega)\right|^{4} d \omega \\
= & \mathbb{E} \int\left|\phi_{e m p}(\omega) \phi_{K}(\omega h)-\phi_{f}(\omega) \phi_{K}(\omega h)+\phi_{f}(\omega) \phi_{K}(\omega h)-\phi_{f}(\omega)\right|^{4} d \omega \\
\leq & C \int \mathbb{E}\left|\phi_{e m p}(\omega)-\phi_{f}(\omega)\right|^{4}\left|\phi_{K}(\omega h)\right|^{4} d \omega+C \int\left|\phi_{K}(\omega h)-1\right|^{4}\left|\phi_{f}(\omega)\right|^{4} d \omega .
\end{aligned}
$$

By Rosenthal's inequality (see Theorem 2.12 in Hall and Heyde (1980)), it is not hard to check that there exists some constant $C$ such that

$$
\mathbb{E}\left|\phi_{\text {emp }}(\omega)-\phi_{f}(\omega)\right|^{4} \leq \frac{C}{n^{2}}
$$

which leads to

$$
\int \mathbb{E}\left|\phi_{e m p}(\omega)-\phi_{f}(\omega)\right|^{4}\left|\phi_{K}(\omega h)\right|^{4} d \omega \leq \frac{C}{n^{2} h} \int\left|\phi_{K}(\omega)\right|^{4} d \omega \leq \frac{C}{n^{2} h} .
$$

It follows from the inequality $\left|\phi_{K}(\omega h)-1\right| \leq 2$ and

$$
\left|\phi_{K}(\omega h)-1\right| \leq \int\left|e^{i \omega h x}-1\right| K(x) d x \leq \omega h \int|x| K(x) d x
$$

that

$$
\int\left|\phi_{K}(\omega h)-1\right|^{4}\left|\phi_{f}(\omega)\right|^{4} d \omega \leq \begin{cases}C h^{4}, & \text { if } \int\left|\omega \phi_{f}(\omega)\right|^{4} d \omega<\infty, \\ C h^{3}, & \text { if } \int|\omega|^{3}\left|\phi_{f}(\omega)\right|^{4} d \omega<\infty \\ C h^{2}, & \text { if } \int|\omega|^{2}\left|\phi_{f}(\omega)\right|^{4} d \omega<\infty \\ C h, & \text { if } \int|\omega|\left|\phi_{f}(\omega)\right|^{4} d \omega<\infty .\end{cases}
$$

By (A.14)-(A.17) we obtain the desired results. 
Proof of Proposition 2. By straightforward calculation and application of Cauchy-Schwarz inequality, we have

$$
\begin{aligned}
\operatorname{Var}\left(\hat{\varphi}_{n}(t)\right) & =\operatorname{Var}\left(\hat{\varphi}_{n}(t)-\varphi(t)\right) \\
& =\operatorname{Var}(\mathrm{I}+\mathrm{II}) \\
& =\operatorname{Var}(\mathrm{I})+\operatorname{Var}(\mathrm{II})+2 \operatorname{Cov}(\mathrm{I}, \mathrm{II}) \\
& \leq \operatorname{Var}(\mathrm{I})+\operatorname{Var}(\mathrm{II})+2 \sqrt{\operatorname{Var}(\mathrm{I}) \operatorname{Var}(\mathrm{II})} \\
& \leq 2 \operatorname{Var}(\mathrm{I})+2 \operatorname{Var}(\mathrm{II}) .
\end{aligned}
$$

It follows from Lemma 5 that

$$
\operatorname{Var}(\mathrm{I})=\frac{\lambda^{2} e^{-2 \lambda t}}{n c^{2}}\left(B_{0}(t)+B_{1}(t)+2 B_{2}(t)\right)+o\left(n^{-1}\right) .
$$

By Lemma 6 we know that the order of $\operatorname{Var}(\mathrm{II})$ is given by (A.13). From these we can easily obtain the results.

Proof of Theorem 1 Firstly, it follows from (A.1) that

$$
\frac{\hat{\varphi}_{n}(t)-\mathbb{E} \hat{\varphi}_{n}(t)}{\sqrt{\operatorname{Var}\left(\hat{\varphi}_{n}(t)\right)}}=\frac{\mathrm{I}-\mathbb{E} \mathrm{I}+\mathrm{II}-\mathbb{E} I \mathrm{I}}{\sqrt{\operatorname{Var}\left(\hat{\varphi}_{n}(t)\right)}} .
$$

It follows from Chebyshev's inequality, Lemma 5 and Lemma 6 that for any $\epsilon>0$, we have

$$
\mathbb{P}\left(\left|\frac{\mathrm{II}-\mathbb{E I I}}{\sqrt{\operatorname{Var}\left(\hat{\varphi}_{n}(t)\right)}}\right|>\epsilon\right) \leq \frac{\operatorname{Var}(\mathrm{II})}{\epsilon^{2} \operatorname{Var}\left(\hat{\varphi}_{n}(t)\right)} \rightarrow 0 .
$$

Then $\frac{\text { II-EII }}{\sqrt{\operatorname{Var}\left(\hat{\varphi}_{n}(t)\right)}}$ converges to zero in probability, and consequently, it can be neglected by Slutsky's theorem. Since $\operatorname{Var}\left(\hat{\varphi}_{n}(t)\right) \sim \operatorname{Var}(\mathrm{I})$ by Proposition 2, it suffices to show that

$$
\frac{\mathrm{I}-\mathbb{E} \mathrm{I}}{\sqrt{\operatorname{Var}(\mathrm{I})}} \stackrel{\mathfrak{D}}{\rightarrow} N(0,1) .
$$

Note that

$$
\mathrm{I}=\frac{1}{n} \frac{\lambda e^{-\lambda t}}{c} \sum_{j=1}^{n} V_{n, j}
$$

where

$$
V_{n, j}=\frac{1}{2 \pi} \int \frac{e^{-i \omega c t}-1+i \omega c t}{(i \omega)^{2}} e^{\lambda t \phi_{f}(\omega)}\left(e^{i \omega X_{j}} \phi_{K}(\omega h)-\phi_{f}(\omega)\right) d \omega .
$$

is a bounded random variable. The asymptotic normality follows by directly checking the sufficient conditions for central limit theorem. This completes the proof.

Proof of Corollary 1. We have

$$
\frac{\hat{\varphi}_{n}(t)-\varphi(t)}{\sqrt{\operatorname{Var}\left(\hat{\varphi}_{n}(t)\right)}}=\frac{\hat{\varphi}_{n}(t)-\mathbb{E} \hat{\varphi}_{n}(t)+\operatorname{Bias}\left(\hat{\varphi}_{n}(t)\right)}{\sqrt{\operatorname{Var}\left(\hat{\varphi}_{n}(t)\right)}}
$$

By Theorem 1, it suffices to show that $\operatorname{Bias}\left(\hat{\varphi}_{n}(t)\right) / \sqrt{\operatorname{Var}\left(\hat{\varphi}_{n}(t)\right)}$ converges to zero, or equivalently, $\sqrt{n} \operatorname{Bias}\left(\hat{\varphi}_{n}(t)\right)$ converges to zero. However, this is obvious thanks to Proposition 1 . This completes the proof. 